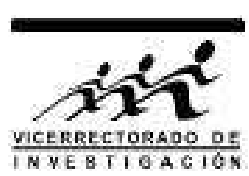

\title{
Dinámica del oscilador de Dirac
}

\author{
Jonatan Vignatti Muñoz y Fulgencio Villegas Silva*
}

Facultad de Ciencias Físicas, Universidad Nacional Mayor de San Marcos, Lima, Perú

Recibido 8 febrero 2016 - Aceptado 28 agosto 2016

\begin{abstract}
Mostramos el campo de Dirac para una partícula masiva libre que describe el movimiento de un fermión relativístico, usando el formalismo de la teoría cuántica de campos. Introducimos una sustitución no-mínima en el operador de momentum, el cual nos produce el oscilador de Dirac, entonces procedemos a cuantizarlo canónicamente y expresamos el operador del campo del oscilador de Dirac en función de los operadores de creación y aniquilación.
\end{abstract}

Palabras claves: Teoría cuántica de campos, fermiones, campo de Dirac.

\section{Dynamic of the Dirac Oscillator}

We show the Dirac field for a free massive particle describing the motion of a relativistic fermion, using the quantum field theory formalism. We introduce a non-minimal substitution on the momentum operator, which gives us the Dirac oscillator so we proceed to quantize canonically and to express the field operator of the Dirac oscillator in terms of creation and annihilation operators.

Keywords: Quantum field theory, fermions, Dirac field.

Desde la primera observación experimental del oscilador de Dirac [1], en 1993, se ha renovado el interés por el estudio de este tema que resulta bastante interesante.

En general, el estudio sistemático del oscilador armónico ha sido la base de las investigaciones en la física teórica, pues la conjetura más importante de nuestros tiempos es considerar el universo como un conjunto de osciladores armónicos, que tienen como formalismo matemático a la maquinaria de la teoría cuántica de campos.

Cualquier potencial se puede aproximar por un potencial armónico en las proximidades del punto de equilibrio. Es la base para la comprensión de los modos complejos de vibración en las grandes moléculas y el movimiento colectivo de los átomos en una red sólida.

Al estudiar la ecuación de Dirac expresando linealmente el momento y las coordenadas, se forma un sistema conocido como el oscilador de Dirac, el cual en el límite no relativístico se convierte en un oscilador armónico con un término de acoplamiento intenso [2].

El oscilador de Dirac consiste en un potencial armónico linealizado, que interactúa con el fermión relativístico.

Desde el punto de vista de la física de partículas, el estudio de este oscilador es de gran importancia para describir el espectro de los mesones, bariones y el confinamiento de los quarks. En este trabajo, analizamos el oscilador de Dirac en el marco de la teoría cuántica de campos con el enfoque divulgativo.

\section{El campo de Dirac}

La ecuación de Dirac para un fermión relativístico tiene la forma,

$$
\left(i \gamma^{\mu} \partial_{\mu}-m\right) \psi=0
$$

donde $m$ es la masa del fermión, $\psi_{\sigma}(x)$, es una función compleja espinorial de 4 componentes, $\sigma=1,2,3,4$ [3]. Esta ecuación es covariante. Se ha considerado el sistema de unidades donde $\hbar=c=1$.

En el tratamiento del campo de Dirac se tiene una densidad lagrangiana invariante de Lorentz, a partir de la cual se deducen las ecuaciones de Dirac, esta lagrangiana es de la forma,

$$
\mathcal{L}=\bar{\psi}\left(i \gamma^{\mu} \partial_{\mu}-m\right) \psi
$$

donde $\bar{\psi}=\psi^{\dagger}(x) \gamma^{0}$, es el espinor adjunto.

Si trabajamos con la densidad lagrangiana anterior para describir la teoría de Dirac, podemos definir la densidad hamiltoniana [7]

$$
\mathcal{H}=-\bar{\psi} \boldsymbol{\gamma} \cdot \nabla \psi+m \bar{\psi} \psi
$$

${ }^{*}$ fvillegass@unmsm.edu.pe 
Usando las relacions de anticonmutación de JordanWigner [7],

$$
\left\{\psi_{\sigma}(x), \psi_{\rho}^{\dagger}\left(x^{\prime}\right)\right\}=\delta_{\sigma \rho} \delta\left(x-x^{\prime}\right),
$$

a partir de donde obtenemos el hamiltoniano del campo de Dirac [7],

$$
H=\int d^{3} x[-\bar{\psi} \gamma \cdot \nabla \psi+m \bar{\psi} \psi]
$$

La ecuación de Heisenberg para el campo $\psi$ está dado por $\dot{\psi}=-i[\psi(x), H]$, con lo cual obtenemos que la ecuación de movimiento del campo de Dirac [4] es

$$
i \dot{\psi}(x)=[-i \boldsymbol{\gamma} \cdot \boldsymbol{\nabla}+m] \psi(x) \text {. }
$$

\section{El oscilador de Dirac}

El efecto de un potencial armónico actuando sobre un fermión relativístico se desarrolla considerando que la ecuación de Dirac corresponde a la linealización de la ecuación de Schrödinger relativística, para el cual el potencial armónico se introduce como su raíz cuadrada, esto es, como un potencial lineal. Dicho sistema es conocido como oscilador de Dirac.

En la interacción del potencial armónico sobre un fermión relativístico de masa $m$, moviéndose en la dirección del eje $z$ [5],

$$
\hat{p_{z}} \rightarrow \hat{p_{z}}-i m \omega \beta \hat{z}
$$

donde $\omega$ es la frecuencia del oscilador.

La ecuación de Dirac para esta interacción, se describe como

$$
i \partial_{0} \psi=\left(\alpha_{3} \cdot\left(\hat{p_{z}}-i m \omega \beta \hat{z}\right)+\beta m\right) \psi
$$

La ecuación anterior toma la forma explícitamente covariante,

$$
\left(i \gamma^{\mu} \partial_{\mu}-m+\sigma^{\mu \nu} F_{\mu \nu}\right) \psi=0
$$

con $\sigma^{\mu \nu}=\frac{i}{2}\left[\gamma^{\mu}, \gamma^{\nu}\right]$, y el tensor asociado con la interacción está dado por

$$
F_{\mu \nu}=\partial_{\mu} A_{\nu}-\partial_{\nu} A_{\mu}
$$

donde se ha definido el cuadripotencial asociado con la interacción como

$$
A_{\mu}=\frac{1}{4}\left(2(u \cdot x) x_{\mu}-x^{2} u_{\mu}\right)
$$

con $u_{\mu}=(m \omega, 0)$ que corresponde a un vector dependiente del marco de referencia.

\section{Acoplamiento $L \cdot S$}

Se observa de la ecuación de Dirac, Ec.(8), que la parte derecha es hermitiana. Además expresando la dependencia de $\psi$ sobre $t$ como $\exp [-i E t / \hbar]$, reescribimos

$$
\psi=\left[\begin{array}{l}
\psi_{1} \\
\psi_{2}
\end{array}\right]
$$

donde $\psi_{1}, \psi_{2}$ son las componentes de la ecuación de onda de Dirac, observando que estas satisfacen las ecuaciones

$$
\begin{aligned}
& \left(E-m c^{2}\right) \psi_{1}=c \boldsymbol{\sigma} \cdot(\boldsymbol{p}+i m \omega \boldsymbol{r}) \psi_{2} \\
& \left(E+m c^{2}\right) \psi_{2}=c \boldsymbol{\sigma} \cdot(\boldsymbol{p}-i m \omega \boldsymbol{r}) \psi_{1}
\end{aligned}
$$

a partir de las cuales se obtiene el acople [6]. Ahora consideraremos $\hbar$ y $c$ en las ecuaciones, con la finalidad de apreciar mejor la intensidad del término de acople

$$
\begin{array}{r}
\left(E^{2}-m^{2} c^{4}\right) \psi_{1}=\left[c^{2}\left(p^{2}+m^{2} \omega^{2} r^{2}\right)-3 \hbar \omega m c^{2}\right. \\
\left.-4 m c^{2}(\omega / \hbar) \boldsymbol{L} \cdot \boldsymbol{S}\right] \psi_{1}
\end{array}
$$

donde

$$
\boldsymbol{L}=\boldsymbol{r} \times \boldsymbol{p} \quad \text { y } \quad \boldsymbol{S}=\frac{\hbar}{2} \boldsymbol{\sigma} .
$$

Escribiendo ahora $E=m c^{2}+\epsilon$, el término $E^{2}-m^{2} c^{4}$ al lado derecho de la Ec.(15) se convierte aproximadamente em ¿n $2 m c^{2} \epsilon$, si $\epsilon \ll m c^{2}$. Así en el límite no relativístico la energía $\epsilon$ del problema se convierte en el autovalor del operador del lado derecho de la Ec.(15) dividido por $2 m c^{2}$, el cual corresponde al hamiltoniano de un oscilador armónico de frecuencia $\omega$ junto con un término de acoplamiento espín-órbita de intensidad $-(2 \omega / \hbar)$.

Debido a este comportamiento en el límite no relativístico, nos referimos a la Ec. (15), como el correspondiente a un oscilador de Dirac, donde el lado derecho conmuta con el momento angular total.

$$
\begin{aligned}
& \boldsymbol{J}=\boldsymbol{L}+\boldsymbol{S} \\
& {[\boldsymbol{J}, \boldsymbol{H}]=0 .}
\end{aligned}
$$

\section{Cuantización canónica del oscilador de Dirac}

Ahora realizamos el tratamiento usual en la teoría cuántica de campos para la cuantización canónica [7]. Primero, se expande el campo en la base definida por las funciones de onda del oscilador de Dirac, teniendo en cuenta que los polinomios de Hermite representan la base para la expansión en series ortogonales y considerando que $n \in \mathbb{Z}$. De esta forma, la expansión en la serie de Fourier del campo 
se escribe como

$$
\begin{aligned}
\hat{\psi}(z, t) & =\sum_{n=-\infty}^{\infty} \hat{b}_{n} \hat{\psi}_{n}(z, t) e^{-i E_{n} t} \\
& =\sum_{n=0}^{\infty} \hat{b}_{+} u_{n}(z) e^{-i E_{n} t}+\sum_{n=1}^{\infty} \hat{b}_{-n} v_{n}(z) e^{i E_{n} t} \\
& =\hat{\psi}_{+}(z, t)+\hat{\psi}_{-}(z, t)
\end{aligned}
$$

donde se han separado las contribuciones de las energías positivas y negativas $\hat{\psi}_{ \pm}(z, t)$, y $u_{n}(z), v_{n}(z)$ son los espinores.

Haciendo uso de las relaciones de anticonmutación, Ec.(4), se puede verificar que los operadores de creación $\hat{b}_{n}^{\dagger}$ y aniquilación $\hat{b}_{n}$ de partículas positivas y negativas, satisfacen la relación de anticonmutación

$$
\left\{\hat{b}_{n}, \hat{b}_{m}^{\dagger}\right\}=\delta_{n, m}
$$

Teniendo en cuenta esta expansión, junto con las propiedades de los polinomios de Hermite, encontramos que el operador hamiltoniano está dado como

$$
\hat{H}=\sum_{-\infty}^{\infty} E_{n} \hat{b}_{n}^{\dagger} \hat{b}_{n}=\sum_{n=0}^{\infty} E_{n} \hat{b}_{n}^{\dagger} \hat{b}_{n}-\sum_{n=1}^{\infty} E_{n} \hat{b}_{-n}^{\dagger} \hat{b}_{-n} .
$$

Usando la relación de conmutación, Ec.(20), se encuentra que

$$
\hat{H}=\sum_{n=0}^{\infty} E_{n} \hat{b}_{n}^{\dagger} \hat{b}_{n}+\sum_{n=1}^{\infty} E_{n}\left(\hat{b}_{-n} \hat{b}_{-n}^{\dagger}-1\right),
$$

por tanto, el operador hamiltoniano sin tener en cuenta la energía divergente del vacío $\sum_{n=1}^{\infty} E_{n}$, se escribe como

$$
\hat{H}=\sum_{n=0}^{\infty} E_{n} \hat{b}_{n}^{\dagger} \hat{b}_{n}+\sum_{n=1}^{\infty} E_{n} \hat{b}_{-n} \hat{b}_{-n}^{\dagger} .
$$

Finalmente, realizamos una transformación canónica sobre los operadores de partículas con energías negativas

$$
\begin{gathered}
\hat{b}_{-n}=\hat{c}_{n}^{\dagger}, \\
\hat{b}_{-n}^{\dagger}=\hat{c}_{n}, \\
\left\{\hat{c}_{n}, \hat{c}_{m}^{\dagger}\right\}=\delta_{n, m},
\end{gathered}
$$

dicha transformación significa que los operadores de aniquilación de las partículas en el mar de Dirac, se interpretan como los operadores de creación de las antipartículas. Con lo anterior, la expansión en serie de Fourier del operador de campo del oscilador de Dirac dada por la Ec.(19), se puede escribir como

$$
\hat{\psi}(z, t)=\sum_{n=0}^{\infty} \hat{b}_{+} u_{n}(z) e^{-i E_{n} t}+\sum_{n=1}^{\infty} \hat{c}_{n}^{\dagger} v_{n}(z) e^{i E_{n} t} .
$$

\section{Aplicaciones}

Se ha aplicado el oscilador de Dirac para un sistema de dos partículas formado por un quark y un antiquark cuyo espectro de autovalores se obtiene diagonalizando el $\mathrm{Ha}$ miltoniano que está dado por

$$
\begin{aligned}
H^{\prime} & =\left(\beta_{1}+\beta_{2}\right)\left(m+\frac{p^{2}}{2 m}-\frac{p^{4}}{8 m}\right) \\
& +V+\frac{1}{4 m^{2}}\left(\mathbf{s}_{\mathbf{1}}+\mathbf{s}_{\mathbf{2}}\right) \cdot[(\mathbf{p} \times \mathbf{E})-(\mathbf{E} \times \mathbf{p})] \\
& +\frac{1}{4 m^{2}} \nabla^{2} V
\end{aligned}
$$

Con el potencial $V=\frac{1}{2} m \omega^{2}\left(\mathbf{r}_{1} \cdot \mathbf{r}_{2}\right)^{2}$. En el centro de masa, con la elección de las componentes de energías positivas el Hamiltoniano se reduce a

$$
\begin{aligned}
H^{\prime} & =\left(2 m+3 \frac{\omega^{2}}{8 m}\right) \\
& +\left(\frac{p^{2}}{m}+\frac{m \omega^{2} r^{2}}{4}+\frac{\omega^{2}}{4 m} \mathbf{L} \cdot \mathbf{S}\right) \\
& -\frac{p^{4}}{4 m^{3}}
\end{aligned}
$$

con $\mathbf{r}, \mathbf{p}$ como coordenada relativa y momentum relativo. El espectro del problema se encuentra diagonalizando la matriz con elementos tales como

$$
\begin{aligned}
& \left\langle n^{\prime} l^{\prime},\left(\frac{1}{2} \frac{1}{2}\right) S ; j, m\left|H^{\prime}\right| n l,\left(\frac{1}{2} \frac{1}{2}\right) S, j, m\right\rangle \\
= & \left(2 m+\frac{3 \omega^{2}}{8 m}+\omega\left(2 n+l+\frac{3}{2}\right)\right) \delta_{n n^{\prime}} \\
+ & \frac{\omega^{2}}{8 m}[j(j+1)-l(l+1)-s(s+1)] \delta_{n n^{\prime}}
\end{aligned}
$$

donde se usa los estados del oscilador armónico de dos partículas con espín, es decir,

$$
\begin{aligned}
& \left|n l,\left(\frac{1}{2} \frac{1}{2}\right) S ; j, m\right\rangle \\
\equiv & \sum_{\mu \sigma}\langle l \mu, S \sigma \mid j m\rangle|n l \mu\rangle\left|\left(\frac{1}{2} \frac{1}{2}\right) S \sigma\right\rangle .
\end{aligned}
$$

Para el el charmonium, las constantes de acoplamiento y la masa en reposo se toman como parámetros ajustables. Para ello, el hamiltoniano efectivo calculado para este sistema está dado por

$$
\begin{aligned}
& H^{\prime}=\left(2 m+\frac{3 \omega^{2}}{8 m}+\omega\left(2 n+l+\frac{3}{2}\right)+\right. \\
&\left.\frac{\omega^{2}}{8 m}[j(j+1)-l(l+1)-s(s+1)]\right) \delta_{n n^{\prime}}- \\
& \frac{1}{4 m^{3}}\left\langle n^{\prime} l^{\prime}\left|p^{4}\right| n l\right\rangle,
\end{aligned}
$$

produciendo los autovalores de energía que se muestran en la tabla 1 junto con los datos experimentales mostrados por Shepherd et al [9]. 


\begin{tabular}{cccllllll}
\hline & & & Exp.(GeV) & Teor. $(\mathrm{GeV})$ & $\mathrm{n}$ & $\mathrm{J}$ & $\mathrm{L}$ & $\mathrm{S}$ \\
\hline $1^{-}$ & $J / \Psi$ & $1 \mathrm{~S}$ & 3.09696 & 3.019815 & 1 & 1 & 0 & 1 \\
$1^{-}$ & $\Psi$ & $2 \mathrm{~S}$ & 4.040 & 4.03981 & 2 & 1 & 0 & 1 \\
$0^{+}$ & $\chi_{c 0}$ & $1 \mathrm{P}$ & 3.41475 & 3.416728 & 1 & 0 & \pm 1 & 1 \\
$1^{+}$ & $\chi_{c 1}$ & $1 \mathrm{P}$ & 3.51066 & 3.47327 & 1 & 1 & 1 & 1 \\
$2^{+}$ & $\chi_{c 2}$ & $1 \mathrm{P}$ & 3.55620 & 3.642902 & 1 & 2 & 1 & 1 \\
\hline
\end{tabular}

los datos experimentales mostrados en [9]

Como se puede observar los valores calculados con el modelo estudiado son bastante próximos a los datos obtenidos experimentalmente para los sistemas ligados de quarks y antiquarks.

\section{Conclusiones}

Se ha cuantizado el oscilador de Dirac siguiendo el tratamiento que se realiza habitualmente para la cuantización canónica de la teoría de Dirac, el operador de campo se representó como una combinación lineal de operadores de creación y aniquiliación, este procedimiento es bastante similar al proceso que se da en el tratamiento habitual para el oscilador armónico cuántico en los textos de mecánica cuántica [10-12].

Se observa que el oscilador de Dirac es un modelo bastante aproximado a los resultados experimentales que muestran los sistemas de quarks-antiquarks.

\section{Referencias}

[1] J. A. Franco-Villafañe, E. Sadurní, S. Barkhofen, U. Kuhl, F. Mortessagne y T. H. Seligman; First experimental realization of the Dirac oscillator, Phys. Rev. Lett. 111, 170405 (2013).

[2] M. Moshinski y A. Szczepaniak The Dirac Oscillator, J. Phys. A: Math. Gen. 22, L817 (1989).

[3] F. Mandl y G. Shaw, Quantum Field Theory, pp.57-58, Wiley and Sons, New York (2010).

[4] W. Greiner, J. Reinhardt, Field Quantization, pp. 123124, Springer-Verlag, Berlín (1996).

[5] M. Moshinsky y Y. Smirnov, The Harmonic Oscillator in Modern Physics, p. 295, Contemporary Concepts in Physics Vol. 9, Harwood Academic, Amsterdam (1996).

[6] R. P. Martínez y Romero, H. N. Nuñez-Yepez y A. L. Salas-Brito, Relativistic quantum mechanics of a Dirac oscillator, Euro. J. Phys. 16, 135 (1995).
[7] Y. Pérez y C. Quimbay, Cuantización canónica del campo del oscilador de Dirac, Rev. Col. Fis. 42(2), 240 (2010).

[8] M. Moshinsky y G. Loyola Barut equation for the particle-antiparticle system with a Dirac oscillator interaction, Foundation of Physics 23(2), 197 (1993).

[9] Matthew R. Shepherd, Jozef J. Dudek y Ryan E. Mitchell; Nature 534, 487 (2016).

[10] Jhon S. Townsend; A moderm approach to Quantum Mechanics, University Science Books, Sausalito, California (2000).

[11] Bipin R. Desai; Quantum Mechanics with Basic Field Theory, Cambridge University Press, Cambridge (2010).

[12] Kurt Gottfried y Tung-Mow Yan; Quantum Mechanics: Fundamentals, Second edition, Springer-Verlag, New York (2004). 